\section{Acute Infections}

Towards the end of the 1931-7 period the sulphonamides were just coming in, with a dosage not fully understood, with some dangers overemphasized and others not realized. When one remembers the horrible problems of septicaemia, arthritis, and osteomyelitis, a total of 25 deaths was not larger than one would expect. In 1947 penicillin was becoming cheaper and more fully available, and in the intervening years its successors have kept appearing with gratifying regularity. In addition, all through this second seven-year period we have had the steady support of readily available blood transfusion-available in quantity to sustain the patient until the specific remedy was found and had time to take effect.

Not only did this disease group almost vanish, to a mortality of one death in a thousand patients, but in the second seven-year period there were double the number of admissions; and an acute infection which in the first period took days, weeks, or even months-for example, in the case of bone diseases - to clear up now suddenly disappears after a few days' treatment with one of the "wonder drugs." This has been accompanied, too, by the almost total disappearance of gross subsequent deformities.

Osteomyelitis suddenly changed; from a septicaemia with simple or multiple local manifestations, it became a disease with a sterile blood stream. The bony lesions still showed necrosis-perhaps a sterile necrosis-till the suitable combination of decompression of the bone by minute drill-holes and the use of an antibiotic was established.

Perhaps another seven-year period 20 years from now may not show as good results if a population of antibioticresistant patients has by then been produced. Or will there still be bigger and better spectra of antibiotics?

\section{Congenital Pyloric Stenosis}

In surgery the best results are obtained from the relief of mechanical obstructions, and this condition is a perfect example. With suitable pre-operative preparation of the patient, with adequate hydration, with a basal narcotic-for example, rectal thiopentone-with the operation performed under a local analgesic, with a transverse incision, and with the abdominal wound closed by a suitably non-absorbent suture-for example, nylon-a simple surgical technique can be evolved. Staining and shock are avoided. The patient should be awake at the end of the operation. There is no fear that the abdominal wound will burst apart, and yet the child is given full freedom. The operation should have a mortality of nil, but the missed diagnosis, the neglected case, and the wasted child with intercurrent disease still appear to make the operation a hazardous procedure, no matter how well carried out.

\section{Summary}

The death rates from some of the more frequent surgical conditions are compared in two seven-year periods, 1931-7 and 1947-53. Some of the factors responsible for this improvement are discussed.

\section{BIBLIOGRAPHY}

British Medical Journal, 1953 2, 1148.

Bunton, G. L. (1953). Ibid., 2, 71. 117.

Kyle, J. (1954). Ulster med. J., 23, 117. Abdominal Surgery of Infancy add, W. E., and Gross, R. E. (1941).

and Childhood. Saunders, Philadelphia.

Children starting school at the age of 5 have, on average, five of their teeth missing, decayed, or filled, said Miss PAT HoRnSBY-SMITH, Parliamentary Secretary to the Ministry of Health, when she opened the new Stroud Green Dental Centre at Croydon on September 28. There was no doubt, she said, that one of the main causes of the increase in dental decay since the end of the war had been the greatly increased consumption of sweets. Children should not eat sweets all day, and teeth should be brushed or the mouth rinsed after they had been eaten. To clean their teeth before retiring and send them to bed with a mouthful of sweets or biscuits was asking for trouble.

\section{MANAGEMENT OF THE PREGNANT DIABETIC*}

BY

\section{J. H. PEEL, F.R.C.S., F.R.C.O.G.}

Obstetrician and Gynaecological Surgeon, King's College Hospital, London

Problems relating to pregnancy in diabetics have in the past two decades aroused a greater interest and achieved a greater importance than the frequency with which pregnancy and diabetes are associated in clinical practice appears to justify. Some years ago Dr. W. G. Oakley and I had occasion to collect information on this subject in Great Britain. We collected details of all the cases treated in 26 different teaching centres, where they tend to concentrate. Over a seven-year period there were only 458 diabetics cared for during pregnancy, which works out at about three cases per annum in each hospital. At my own hospital, King's College Hospital, we have a very large diabetic clinic under the direction of Drs. R. D. Lawrence and W. G. Oakicy. We have therefore a great concentration of clinical material, and for many years now I have looked after 20-30 cases a year. I shall quote from my personal experience of a series of 298 pregnancies in diabetic women seen during the last 11 years.

Why are we, as obstetricians, taking a so much greater interest in this subject? I think it is because in this relatively small group of patients we encounter problems relating to foetal size, placental function, intrauterine foetal death, hydramnios, toxaemia, congenital foetal abnormalities, and neonatal mortality. The solution of any or all of these problems in the diabetic will, we hope, help us to understand more about foetal physiology and pathology in general. But knowledge is slow to accumulate, and there are many riddles still awaiting elucidation before we can explain fully why for the diabetic woman reproduction is such a relatively unproductive enterprise.

The control of maternal diabetes by means of insulin and dietary measures has reduced enormously the maternal risks during pregnancy. The maternal mortality is now as little as $1 \%$ or less- $0.4 \%$ Joslin (1946) -compared with $45 \%$ quoted by Whitridge Williams (1909) 45 years ago. Yet such figures tend to disguise the seriousness of pregnancy as an episode in the life of the diabetic. We have become accustomed to seeing these patients so cared for and their diabetic lives so organized that we fail to appreciate how closely they often come to disaster for themselves as well as their babies owing to the profound metabolic and endocrinal changes that accompany pregnancy. The hazards of repeated pregnancies in the diabetic in terms of morbidity are on the whole under-emphasized. Good control of diabetes throughout pregnancy can reduce the foetal mortality, as Lawrence and Oaklèy (1942) showed, but cannot abolish it.

Foetal mortality can be considered in relation to avoidable and unavoidable factors, the most important avoidable factor being control and supervision of maternal diabetes. But there are still unavoidable factors which make it impossible to reduce the foetal loss much below $20 \%$. The last ten years have seen a

*Read in the Section of Obstetrics and Gynaecology at the Joint Annual Meeting of the British Medical Association, Canadian Medical Association, and Ontario Medical Association, Toronto, 1955. 
considerable number of papers, each quoting from a relatively small series of cases, and each endeavouring to produce a lower foetal mortality than the other. These figures have, however, a limited comparative significance, if for no other reason than the lack of information given about the selection of cases and the indications for therapeutic termination. But they all emphasize a depressingly high foetal mortality-my own is $27 \%$ in the present series of 298 pregnancies. In marked contrast the $10 \%$ foetal loss quoted by Nelson, Gillespie, and White (1953) is a challenge to all those working in this field, and it is well known that they attribute their excellent results largely to hormone therapy. This subject is referred to again later.

\section{The Pre-Diabetic State}

It is now well established that married women who develop diabetes in middle life give obstetric histories very similar to those of the long-established diabetic. Peel and Oakley (1949) found a $23 \%$ foetal loss and a $27 \%$ incidence of babies weighing more than $10 \mathrm{lb}$. $(4.5 \mathrm{~kg}$.) in 237 prediabetic pregnancies, and other authors have quoted similar figures. There is, of course, very great theoretical significance in these facts, which suggest that the large babies and high foetal loss are not directly related to disordered carbohydrate metabolism of the established diabetic as such. It is thought that there may be a close relationship between the diabetogenic and growth factors of the anterior pituitary gland, and, further, that there is an overactivity of these for many years before the onset of clinical diabetes. It has been suggested that the pre-diabetic father may be a responsible agent in the production of giant babies, although it has not been possible to implicate him as a cause for high foetal loss.

This work has yet to be confirmed, but is mentioned because of its theoretical interest in relation to hereditary and genetic factors in these cases. The practical significance of these facts is simply that if we encounter pregnant women who give birth to very large babies of $10 \mathrm{lb}$. $(4.5 \mathrm{~kg}$.) and over or who have a series of obstetric disasters due to stillbirth or early neonatal death, otherwise unexplained, it is important to investigate them as potential diabetics. A tendency towards adiposity may be noticeable and an abnormal glucose-tolerance curve discovered.

\section{Diabetes Developing During or Shortly After Pregnancy}

It is well known that pregnancy may affect carbohydrate metabolism. Apart from a lowered renal threshold which may account for the appearance of glycosuria, there may be a decrease in glucose tolerance. This may be due to the increased production of adrenal cortical hormones which occurs in pregnancy. The normal person's pancreatic islets are able to adapt rapidly to this increased mobilization of glucose, so that hyperglycaemia is only transient. In the patient with a diabetic diathesis, however, repeated pregnancies may put a severe strain upon the pancreatic islets, which then fail to adapt themselves. One may, on the one hand, encounter the patient who in succeeding pregnancies is suspected of diabetes because of persistent glycosuria and a glucose-tolerance curve which, while resembling the true diabetic curve, returns to normal as soon as pregnancy is over. On the other hand, a certain number of these patients do not in point of fact recover normal carbohydrate metabolism and become established diabetics requiring insulin as well as dietary control within a few months of delivery. Viewed from this aspect, pregnancy may be a precipitating factor in the development of clinically manifest diabetes. From the practical aspect of this problem it is essential to investigate persistent glycosuria in pregnancy not only during the pregnancy but for some time afterwards. It is equally important to follow up the patient who has delivered a large stillborn baby without other explanation and to investigate carefully the family history.

\section{Pregnancy in the Established Diabetic}

The problem presents itself most frequently in this way. The established diabetic seeks medical advice concerning the prospects of a successful pregnancy or she may be already pregnant. The literature is very sparse relating to the principles upon which either these patients should be encouraged to continue their pregnancy or therapeutic termination be advised. To give exact and rule-of-thumb indications for therapeutic termination is always difficult, if not impossible, for any pre-existing medical reason, and the same applies to the diabetic. But I am sure that if good results are to be obtained from the point of view of both the health and the well-being of the mother and of foetal survival a greater degree of selection is necessary than we have often carried out in the past. The following factors should be considered in every case.

1. Family History.-Whilst exact figures are still not available, no one would deny, on the clinical evidence we have, that a strong family history is obtainable in a large number of diabetic patients. What percentage of the babies born to diabetic mothers will ultimately become diabetic we do not yet know, and it will probably be another 25 years before we can have a sufficiently long-term follow-up of these babies to know the answer. But a strong family history of diabetes, especially if both father and mother are diabetics and have diabetic family histories, is a strong indication for not encouraging further reproduction.

2. Vascular Complications of Diabetes.-These are well known, and Nelson et al. divide their patients into six groups, of whom the last three-D, E, and F-show vascular complications of diabetes. From the viewpoint of foetal survival it is not possible to relate foetal loss directly with duration or severity of diabetes, but the presence of marked hypertension and albuminuria renders the prognosis very poor. As with nypertension and nephritis in the non-diabetic, an unexpectedly favourable outcome to a particular pregnancy may be achieved on occasion with careful management, but this does not alter the generally poor foetal prognosis in such cases. The increased hazards to the mother from superimposed toxaenaia, accidental haemorrhage, retinitis, and renal failure are obvious.

3. Obstetric History.-There is no doubt that there exists an entity we sometimes call a bad obstetric history. It is often difficult to dissociate a bad obstetric history from bad luck and bad management, in this as in all bad obstetric histories. There is equally no doubt, however, that these recurrent obstetric disasters do tend to be repeated in the individual patient. We find that approximately $50 \%$ of our multiparous diabetics who lose their babies have already lost one or more in previous pregnancies. A recent obstetric disaster in a patient refusing termination illustrated the point. The patient, pregnant for the fifth time, with a mild hypertension, developed an acute toxaemic ante-partum haemorrhage at 34 weeks. Her previous four pregnancies had yielded two live and two dead babies. Such histories are not difficult to reproduce from our records. A further point of importance from this aspect of the subject is the tendency for congenital abnormalities of the babies to reproduce themselves in succeeding pregnancies.

4. Availability of Expert Medical Care and the Co-operation of the Patient.-There is little doubt that the most important factors concerned in the care of the pregnant diabetic patient are the availability of constant expert medical supervision and control of the diabetic condition throughout the pregnancy. An unintelligent and uncooperative patient may endanger not only the baby's life but also her own.

5. Size of Family.-Opinions may differ on this point, but the diabetic woman whose life is inevitably modified and limited to some extent by her disease is seldom a suitable person to have a large family, apart from the deleterious effects of repeated pregnancies upon her diabetic condition and the fact that the diabetic patient is not the most suitable for reproduction from the genetic standpoint. These considerations are debatable, however, and will not be further elaborated here.

A very careful assessment is therefore necessary at the beginning of pregnancy, and if good results are to be obtained the closest co-operation between a physician experienced in the management of diabetes as well as a good obstetric team is an essential. As an obstetrician I do not feel fully competent to deal with the detailed management of the patient from the point of view of dietary control and insulin requirements. I merely stress the difficulties that tend to arise from a lowered renal threshold, the 
tendency to vomiting, and ketosis inherent in the pregnant state, which make the careful control of blood-sugar levels and diabetic state in general often very difficult. There is a tendency to instability, especially in the early weeks, if vomiting is present, and again about 20-24 weeks. It is surprising how often the baby survives repeated attacks of ketosis or hypoglycaemia ; but the more frequent and severe they are the more must the foetal prognosis inevitably worsen. The requirements of insulin usually rise during pregnancy and fall during labour and the early puerperium. This latter is of special practical importance, because a continuation of the pregnancy level of insulin dosage after delivery may soon lead to dangerous hypoglycaemia unless the true condition is recognized and glucose, not insulin, given.

I now pass to aspects of the problem which are more particularly obstetric.

\section{Effects of Diabetes on the Pregnancy}

The effects during the first two trimesters of pregnancy are minimal. I know of no convincing evidence of an increased incidence of abortion in diabetics. They are particularly prone to pyelitis and to monilial vulvovaginitis. The latter, whilst being impossible to cure during pregnancy, can be kept under control by the usual methods of local treatment. I have found painting with gentian violet $1 \%$ and the daily use of " pruvagol" vaginal cream the most useful remedy. It is after the 28th week of pregnancy that the main obstetric complications arise - the rapid growth of the foetus, often associated with hydramnios, the development of preeclamptic toxaemia, and intrauterine foetal death. Cardell (1953) brought forward evidence to show that babies of less than 28 weeks gestational age are not larger than those of non-diabetic mothers. The factor, whatever it is, causing the giant baby is operative mainly in the last three months of pregnancy. The same applies to the development of hydramnios. Whilst we know that there is an excess of sugar in the amniotic fluid of the diabetic patient, there is no direct relation between the amniotic sugar level, the maternal or foetal blood-sugar levels, and the amount of liquor amnii (Peel and Oakley, 1949).

Pedersen and Jorgensen (1954) have shown a higher incidence of hydramnios and a greater amount of fluid in a series of cases treated for only a short time compared with a series treated throughout their pregnancies and hospitalized during the 30 days before delivery. They were, however, unable to deduce which part of the treatment given was the effective agent in reducing the incidence of hydramnios. Personally I have found no method of control of the development of hydramnios of any value, and abdominal paracentesis as a measure for reducing the fluid temporarily is also of very doubtful value. In my experience it is apt to cause intrauterine death within a few days, however slowly the liquor is withdrawn. Pedersen and Jorgensen's treatment did not include hormones, mercurial diuretics, and ammonium chloride, all of which are used by Nelson et al. (1953) and given pride of place as therapeutic agents used to decrease the incidence of all the obstetric complications in the last three months of pregnancy. The incidence of preeclamptic toxaemia is higher than in non-diabetic pregnancies, but the factual incidence varies with the extent to which premature termination is practised.

The incidence of toxaemia in the present series is $13 \%$, but figures varying from 3 to $50 \%$ have been quoted. The development of both or either of these complications (and there is an undoubted association between the two) is an event of supreme importance. From the foetal point of view the development of marked hydramnios is more serious than toxaemia, because it so often heralds intrauterine foetal death.

Much has been written about the factors responsible for the high foetal loss in diabetic pregnancy, and some of the possible factors are briefly mentioned:

1. Severity of Diabetes.-There is, no relation between the severity of diabetes, as judged by insulin needs, and foetal loss.
2. Age of Onset of Diabetes.-Oakley (1953) has shown that there is no relation between foetal loss and age of onset of diabetes (Table I).

TABLE I.-Relation of Foetal Mortality to Age of Onset of Maternal Diabetes (Oakley, 1953)

\begin{tabular}{|c|c|c|}
\hline $\begin{array}{l}\text { Age of Onset } \\
\text { of Diabetes }\end{array}$ & $\begin{array}{l}\text { No. of } \\
\text { Babies }\end{array}$ & $\begin{array}{c}\text { Foetal } \\
\text { Mortality }\end{array}$ \\
\hline $\begin{array}{ll}1-15 & . \\
16-24 & .\end{array}$ & $\begin{array}{r}70 \\
105 \\
92\end{array}$ & $\begin{array}{l}21(30 \%) \\
24(23 \%) \\
29(31 \%)\end{array}$ \\
\hline
\end{tabular}

3. Duration of Diabetes.-Oakley has also shown that there is no relation between foetal loss and duration of diabetes (Table II).

TABLE II.-Relation of Foetal Mortality to Duration of Maternal Diabetes (Oakley, 1953)

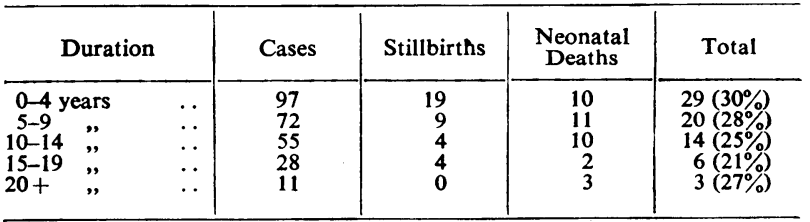

4. Toxaemia.-The foetal loss in toxaemic cases in the present series is $44 \%$-definitely higher than in the nontoxaemic cases.

5. Hydramnios. - It is almost impossible to obtain the exact incidence of this from the literature and, indeed, from my own cases. Some excess liquor is present in nearly all diabetic patients, so that it is most difficult to decide what constitutes hydramnios. Its development fairly acutely between 30 and 36 weeks is always, in my experience, a bad prognostic sign and indicates the need for early termination.

The exact and immediate cause of intrauterine death has not yet been determined. Its incidence steadily rises as the pregnancy advances towards term (Peel and Oakley, 1949), owing presumably to placental insufficiency. One imagines the rapidly growing and pathologically large baby outgrowing the supply of oxygen which can reach it through the placenta. There are two pieces of evidence which suggest the explanation of intrauterine death, and at present we are carrying out investigations along these lines. During the course of a prolonged clinical trial of hormone therapy, an extensive series of hormone assays has been carried out by Professor C. H. Gray at King's College Hospital. The results of these assays have been subjected to critical and statistical analysis and will be published in due course. The results so far suggest that if perinatal death is impending an abrupt and progressive drop in the excretion of pregnanediol and allied chemical compounds in the urine occurs between the 30th and 32nd weeks of pregnancy. This is presumably an indication of failing placental function, but it suggests the possible value of urinary pregnanediol estimations from the prognostic point of view even though the biochemists are not yet completely satisfied with the specific method of estimating this substance or its precise relation to progesterone metabolism.

We are at present carrying out this test weekly from the 28th week onwards, and look upon a progressively falling excretion after the 32nd week as evidence of impending intrauterine death. The number of cases on which we have so far carried out this investigation is not yet large enough to enable us to draw a firm conclusion of its value. Another investigation at present being carried out in our department is an estimation of the cord haemoglobin of the baby at birth and also the oxygen saturation in the blood. The work of Walker (1954) in relation to the post-mature foetus has shown that the baby compensates for a falling oxygen saturation due to placental insufficiency by a steady increase in the haemoglobin level, and that therefore the foetal haemoglobin level steadily increases up to the time of delivery. We are finding haemoglobin levels in the diabetic 
babies delivered at 36 weeks as high as we would expect at term, but again I cannot yet draw final conclusions until we have a sufficiently large series.

White's claim that hormone therapy minimizes the overall foetal loss has been doubted by many authors in Great Britain. The Medical Research Council has organized a clinical trial of hormone therapy, employing both stilboestrol and ethisterone in doses shown in Table III.

TABLE III.-Schedule of Daily Hormone Dosage

\begin{tabular}{cc|c|c}
\hline \multicolumn{2}{c|}{ Stage of Pregnancy } & Stilboestrol (mg.) & Ethisterone (mg.) \\
\hline Up to the end of the 19th week. & 50 & 25 \\
20th-23rd weeks inclusive &. & 100 & 50 \\
24th-27th \# \#\# & $\cdots$ & 100 & 75 \\
28th-31st \#" & 150 & 125 \\
32nd week until delivery &. & 200 & 250 \\
\hline
\end{tabular}

We ourselves have treated 56 patients with hormones according to this schedule : 35 babies survived, 21 were lost. Compared with a control series there was no alteration in the incidence of oedema, albuminuria, toxaemia, or hydramnios, and no difference in the birth weights. The more comprehensive figures from the Medical Research Council include eight other centres besides our own. They bear out our own findings and are in process of publication.

It appears from our investigations that, although hormone therapy has no effect upon foetal survival, it does affect the level of hormone excretion. It does not, however, prevent the drop in the excretion of pregnanediol and allied chemical compounds in the urine that occurs after the 32nd week in cases where foetal death is impending. This is really what one would expect on theoretical grounds. The altered hormone balance is the result of failing placental function and not the cause of it. Substitution therapy apparently corrects the hormone imbalance as judged by excretion studies, but has no influence upon the physiological function of the placenta and so has no effect whatsoever upon foetal survival.

\section{Mode and Time of Delivery}

If pregnancy is allowed to continue to term intrauterine death may occur. Some clinicians allow pregnancy to continue until the onset of spontaneous labour, in the belief that premature termination, whilst preventing intrauterine death, does not lower the overall foetal loss, because of an increased incidence of neonatal death due to prematurity. I have always favoured premature termination, however, in spite of a high incidence of neonatal death. Other factors than prematurity come into the causation of neonatal death, and we hope for a steady improvement in foetal survival, whereas at present there is little that we can do to prevent intrauterine death. For some years, whilst the hormone therapy trial was being conducted, we decided to standardize obstetric management as much as possible. In consequence we carried out caesarean section at the 37 th week in all primiparae and in a great many multiparae. Until antenatal treatment can effectively lower the incidence of intrauterine death and foetal size, caesarean section must inevitably remain the most suitable method of termination. Following artificial rupture of the membranes, there is often a long interval before the onset of labour, especially in the primipara, and this may be followed by inertia in the first stage. During all this time difficulties may arise in controlling the diabetic condition of the mother, with the result that foetal distress may occur. Therefore even after induction caesarean section may become necessary. Nevertheless I think one should exercise judgment about the best method of termination. In the presence of hydramnios, toxaemia, a very large baby, or a very unfavourable cervix, caesarean section is, I think, best, especially if termination is necessary before 36 weeks. On the other hand, in multiparae with a satisfactory previous obstetric history and in some primiparae, where the baby is not unduly large, where hydramnios is minimal and the cervix favourable, induction should be employed, especially if it has been thought safe to allow the pregnancy to proceed to the 38 th week. It has always. been my experience that neonatal death was lower in cases delivered vaginally. Our neonatal death rate is $18.5 \%$ in 199 caesarean sections. Our induction number is small by comparison, but out of 39 patients induced (the baby being alive at the time of induction) 9 babies were lost, but 4 of these died during labour, so that the neonatal death rate was $12 \%$. These figures are not of statistical significance, but they suggest, at any rate, that mode of delivery has little to do with neonatal death. Against induction are, of course, the hazards of a long labour and the difficulties in the vaginal delivery of a very large baby. Very careful judgment is necessary, therefore, in selecting cases suitable for induction.

The majority of babies that die in the neonatal period die within 48 hours of birth. The incidence of congenital abnormalities in our series is $8 \%$, but Cardell has shown that their incidence in post-mortem material is not higher than normal, indicating that congenital abnormalities are not an important cause of neonatal death. Equally $I$ have shown that hypoglycaemia is not an important cause. We have found, however, that in $85 \%$ of our babies dying in the neonatal period hyaline membrane disease is demonstrable. Space does not permit further discussion upon this interesting condition, which is known to occur predominantly but by no means exclusively in premature babies delivered by caesarean section, but it indicates the main lines upon which the newborn baby must be treated. Reutter (1954) suggests that intrauterine anoxia may be a predisposing factor in the subsequent development of hyaline membrane after birth. This would certainly lend support to the conception that the very babies that die in the neonatal perioa are the ones that would have died in utero from anoxia had they not been delivered.

Again space does not permit of a detailed discussion concerning the management of the newborn of the diabetic mother. Suffice it to say that the first 24 hours are the most critical ; the maintenance of a good airway and gastric suction are vital; the administration of oxygen is invaluable; also, the nurse must be experienced in the care of the premature baby. I have little experience yet of the value of corticotrophin (A.C.T.H.) and cortisone or the administration of potassium to the baby. There are theoretical considerations suggestive of the possible value of this, and preliminary investigations are being undertaken in my department. In this subject there is a rich field for speculation and research, and I have been able merely to touch on the main problems involved and indicate the lines upon which we have been working and upon which we intend to proceed.

\section{Summary}

The clinical association of diabetes mellitus and pregnancy is still comparatively uncommon, though increasing. The obstetrical interest lies in relation to problems of foetal size, placental function, intrauterine and neonatal death, hydramnios, toxaemia, and congenital foetal abnormalities in general.

The overall foetal mortality remains high.

Three groups are briefly discussed: $(a)$ the prediabetic; $(b)$ diabetes developing during or soon after pregnancy ; $(c)$ pregnancy in the established diabetic.

The indications for therapeutic termination are considered.

The main problems during the antenatal period arise during the last trimester, when there is an especial tendency for the development of hydramnios and toxaemia, and for intrauterine death to occur.

The high foetal loss is considered in relation to severity, age of onset, and duration of diabetes, and to hydramnios and toxaemia.

The results of a clinical trial using oral hormones (stilboestrol and ethisterone) are given, suggesting that they have no effect upon foetal survival. 
Brief reference is given to hormone assays which have been carried out at King's College Hospital, and their possible prognostic significance indicated.

Premature termination of pregnancy before the end of the 37 th week is recommended, and the relative places of caesarean section and induction of labour are discussed.

The importance of hyaline membrane disease as a cause of neonatal death and its possible relation to intrauterine anoxia are explained.

\section{REFERENCES}

Cardell, B. S. (1953). J. Obstet. Gynaec. Brit. Emp.. 60, 834

Joslin, E. P. (1946). Treatment of Diabetes Meilitus. London.

Lawrence, R. D., and Oakley, W. G. (1942). Quart. J. Med. 11, 45.

Nelson, H. B., Gillespie, L., and White, P. (1953). Obstet. and Gynec., 1, 2 i 9

Oakley, W. G. (1953). British Medical Journal, 1, 1413.

Pedersen. J., and Jorgensen, G (1954).Acta endocr. (Kbh), 15, 333.

Peel, J. H., and Oakley, W. G. (1949). 12th British Congress of Obstetrics and Gynaecology, p. 161

Reutter, F. (1954). Gynaecologia (Basel), 137, 367

Walker, J. (1954). J. Obstet. Gynaec. Brit. Emp., 61, 162.

\section{CLINICAL ASPECTS OF ATYPICAL CORONARY DISEASE* BY}

\author{
KENNETH HARRIS, M.D., F.R.C.P. \\ Senior Physician and Cardiologist, University College \\ Hospital, London
}

It is generally agreed that more cases of coronary arterial disease are being recognized to-day than formerly. We know from Ryle and Russell (1949) that in Great Britain there has been an increase in the mortality from coronary disease in the post-war years, but the increase is less rapid than in the pre-war years. This increased mortality has occurred in spite of improved methods of treatment by anticoagulants, etc. Early and accurate diagnosis is therefore of fundamental importance, in order that the treatment can be started as soon as possible.

I propose to deal with atypical angina pectoris and with some of the clinical aspects of cardiac infarction.

\section{Angina Pectoris}

First let us remember that it was William Heberden who was the first to give, in 1768 , a clear and accurate description of angina in a paper entitled, "Some Account of a Disorder of the Breast." In this paper Heberden showed that angina pectoris may occur with no physical signs and with an apparently normal cardiovascular system, and this has been recognized ever since his time. A diagnosis often has to be made on the history obtained from a patient.

The familiar picture of angina pectoris is that of pain in the centre of the chest radiating down into the left arm to the wrist. It is less well known, however, that the pain may radiate in other directions, or the case may present itself with different symptoms. In a series of cases investigated by me during a five-year period, pain extended from the chest into both arms in $17 \%$ of the cases.

In the "reversed" type the pain in all respects follows the classical pattern of angina pectoris, except for the distribution and radiation, which is the reverse of the usual. The patient complains that the pain starts in his left hand, wrist, or arm, or the left side of his neck, and radiates up into his chest, and is associated with the usual feeling of constriction in the chest.

Next, there is the patient who complains of a sensation of a band round his wrists or arms. On careful inquiry he may admit that it sometimes amounts to a pain; he may

* Read in the Section of Medicine at the Joint Annual Meeting of the British Medical Association, Canadian Medical Association, and Ontario Medical Association, Toronto, 1955. not mention, unless asked, that it is directly relafed to exertion or is accompanied by a retrosternal pain. He may even have been treated as a case of neuritis or myofibrositis before a cardiac cause for his symptoms was suspected.

Quite a number of my cases have complained of a pain in the jaws or gums. One man said that he could not wear his dentures after a meal because of this pain; another man said that his jaws "ache at times."

It will be remembered that the word "angina " does not mean pain, but a strangling. There are the patients whose main concern is the choking or strangling sensation in the throat. An interesting example of this was a patient who reported to my out-patient clinic two years ago, and gave a history of a curious choking sensation in his neck, saying that he felt he was "breathing in cold air." This had been present for two months, and occurred only on walking; it had not interfered with his work, but he had noticed a pain across his chest, with slight dyspnoea, on exertion. At that tıme a diagnosis of atypical angina had been made. Two years later he gave a classical history of angina with considerable incapacity.

Several examples of angina have been sent to my clinic by doctors because they have been worried by the patient's complaint that the pain is worse after meals and they suspected some form of dyspepsia. A recent example of this was a carpenter, who found that his capacity for work was curtailed by his midday meal; he stated that he could do four times as much work in the afternoon if he went without his lunch. A full and carefully taken history revealed that this limitation of work was due to angina, which followed on slight exertion after a heavy meal.

The details of another patient, who experienced pain in his phantom hand, are of special interest. Two such cases have also been recorded by Sir Henry Cohen and Wallace Jones (1943). The man, aged 58, had had a gunshot wound of his left elbow in the 1914-18 war. After excision of the joint, sinuses and sequestra developed, so that amputation above the elbow had to be performed. Three months after the operation angina of effort developed; the patient complained that the pain radiated into the elbow and all the fingers of his phantom hand. When, six months later, he had an attack of coronary thrombosis, the pain followed a similar distribution in the phantom hand.

If young patients develop angina without any obvious cardiovascular disease the possibility of hypercholesterolaemia may be considered, although it is a very rare condition. I have recently seen two members of one family in which this interesting rarity existed. A Cypriot waiter, aged 24 , attended my clinic complaining of severe angina pectoris of three months' duration. Since the age of 12 he had had yellow nodules in the skin (xanthomata). An electrocardiogram was normal, but after an exercise test it showed the classical E.C.G. changes of angina (depressed ST segments, etc.). His blood cholesterol was $650 \mathrm{mg}$. per $100 \mathrm{ml}$. His angina was considered to be due to deposits of cholesterol in his coronary arteries, and to be part of the general disease known as xanthomatosis tuberosum multiplex. He died two years later of cardiac infarction. His sister, aged 30, came to my clinic about this time; she had had skin nodules for eight years and angina pectoris for three years. Her blood cholesterol was $860 \mathrm{mg}$. per $100 \mathrm{ml}$. She died suddenly about one year later, whilst out shopping, as a result of coronary thrombosis.

These are some of the unusual types of angina. How, then, can we make a diagnosis in an atypical case ? The answer is simple; it is by a most scrupulously taken history. Firstly, the method in which the patient gives his history is of great value ; his whole manner of presentation is convincing, since he has no need to use the florid and exaggerated phrases that are so often associated with patients suffering from the neuroses. The patient is usually intelligent, and gives a definite history, showing how handicapped he now is, whereas previously he was a fit man. Moreover, there is an absence of inconsistencies in his history; there may be variations in the amount of exciting exercise. but 